A N N A L E S Annales de Bretagne et des Pays de l'Ouest

Anjou. Maine. Poitou-Charente. Touraine

109-4 | 2002

Les étrangers dans l'Ouest de la France (XVIII ${ }^{\mathrm{e}} \mathrm{XX} \mathrm{e}^{\mathrm{e}}$ siècle)

\title{
De Beni Ayatt à Angers, mémoire et itinéraire d'une communauté berbère du Moyen Atlas marocain (1960-2000)
}

\section{Chadia Arab}

\section{OpenEdition}

\section{Journals}

Édition électronique

URL : http://journals.openedition.org/abpo/1536

DOI : 10.4000/abpo.1536

ISBN : 978-2-7535-1488-1

ISSN : 2108-6443

Éditeur

Presses universitaires de Rennes

Édition imprimée

Date de publication : 20 décembre 2002

Pagination : 231-240

ISBN : 978-2-86847-794-1

ISSN : 0399-0826

Référence électronique

Chadia Arab, « De Beni Ayatt à Angers, mémoire et itinéraire d'une communauté berbère du Moyen Atlas marocain (1960-2000) ", Annales de Bretagne et des Pays de l'Ouest [En ligne], 109-4 | 2002, mis en ligne le 20 décembre 2004, consulté le 02 mai 2019. URL : http://journals.openedition.org/ abpo/1536; DOI : 10.4000/abpo.1536 


\title{
De Beni Ayatt à Angers, mémoire et itinéraire d'une communauté berbère du Moyen Atlas marocain (1960-2000)
}

\author{
Chadia ARAB \\ Doctorante en géographie, Université de Poitiers
}

\begin{abstract}
Nous présentons ici une filière migratoire marocaine qui s'est constituée dans les années 1960 sur Angers ${ }^{1}$. L'intérêt de cette étude vient des faibles taux d'immigration et d'émigration dans les deux espaces de départ et d'arrivée; il s'agit de déterminer pourquoi la population migrante de Beni Ayatt a choisi de s'installer en majorité sur Angers. Quand les premières générations sont arrivées en France, c'était pour améliorer leurs conditions de vie. Ainsi, elles pouvaient ensuite repartir au pays pour y investir leurs économies. Aujourd'hui, ces hommes ont entre 50 et 70 ans environ, une bonne partie d'entre eux est à la retraite ou en préretraite. Leur désir de retour est fort mais l'échéance est toujours repoussée à une date indéterminée.
\end{abstract}

Comment cette population qui a quitté Beni Ayatt dans les années 1960 pour s'installer sur Angers, s'inscrit-elle actuellement sur ces deux espaces géographiques et répond-elle à la dualité de ces espaces? Nous tenterons de répondre à cette problématique en étudiant trois points : comment s'est réalisée l'installation de la communauté sur Angers? Comment la population s'intègre-t-elle à l'espace angevin? Comment l'espace d'origine est-il investi par les migrants? Avant d'aller plus loin, une présentation de la population et l'espace d'étude est nécessaire.

Beni Ayatt, commune marocaine dont sont originaires plus de 400 personnes vivant à Angers

\section{La population d'étude dans la ville d'Angers}

Aujourd'hui, la proportion d'étrangers présents en France serait de $5 \%$ et s'expliquerait à la fois par la mise en place d'une législation "rigoureuse "

1. Cette contribution reprend les principaux résultats obtenus lors de nos travaux de maîtrise à l'Université d'Angers et de DEA à l'Université de Poitiers. Thèse en cours : Des anciennes filières aux nouvelles stratégies migratoires : l'exemple des Beni Mellal au Maroc. 
(740000 entrées en 1977 contre 110000 en 1992), et par une croissance du nombre d'acquisitions de la nationalité (110000 en 1996). En Maine-et-Loire, on dénombre 16000 étrangers sur une population de 722600 habitants soit 2,2 \% de la population. Les Maghrébins représentent $37 \%$, dont la majorité sont des Marocains, 31 \% sont des Européens, 14 \% des Asiatiques et $4 \%$ viennent de l'Afrique noire. Il existe trois communes du département où la population étrangère est importante : Angers avec 5400 étrangers soit 3,7 \% de la population, Cholet avec 4800 étrangers soit 8,5\% de la population, Trélazé avec 850 étrangers soit $8 \%$ de la population. À Angers, $70 \%$ des étrangers vivent dans les quartiers de Monplaisir, la Roseraie et Belle-Beille. En 1990, 660 étrangers vivant à Angers venaient de pays de la CEE (Europe des 12) dont 40 Italiens, 148 Espagnols et 212 Portugais. On comptait en outre 272 Turcs, 276 Algériens, 644 Tunisiens et 1812 Marocains qui constituent de loin la première communauté étrangère présente dans la ville.

Au départ, la population concernée par cette étude est constituée des 28 hommes provenant de la commune de Beni Ayatt et qui s'installent à Angers dans les années 1960. Puis dans les années 1970, par des regroupements familiaux, les femmes et les enfants les ont rejoints. Ainsi, 56 primo-migrants (28 hommes et leurs femmes) sont arrivés à Angers dans les années 1960 et 1970. Puis, nous pouvons compter 211 enfants dans la seconde génération dont certains sont déjà des adultes et ont pu fonder des familles. Parmi ces 211 personnes, 67 sont nées au Maroc et sont venues avec leurs mères dans les années 1970, et 144 sont des Français issus de l'immigration. Enfin, la troisième génération représente 138 personnes. Depuis quelques années une nouvelle migration se produit, elle est le fait de 7 anciens combattants. Ce qui donne un effectif total de la population étudiée de 412 personnes.

\section{Comment s'est formé ce champ migratoire Beni Ayatt/Angers?}

Nous avons pu reconstituer la chaîne migratoire de la communauté de Beni Ayatt grâce aux différents entretiens réalisés avec les immigrés de la première génération, et particulièrement les plus âgés. Monsieur AS. L. est le premier arrivant de la communauté de Beni Ayatt. Nous avons pu le rencontrer lors de notre travail sur le terrain au Maroc. Il a actuellement 61 ans et est à la retraite. Son témoignage permet de comprendre l'histoire de cette installation :

« Je suis arrivé en France le 21 mars 1965 [...]. J'avais eu un contrat de travail pour travailler dans le Canal du Nord, une société qui travaillait avec le barrage d'Afourer [commune limitrophe à Beni Ayatt par l'Est]. Le contrat a été envoyé par la France. Ce n'était pas encore à Angers, mais du côté de la frontière allemande, vers l'Alsace, c'est là qu'il voulait m'envoyer. J'ai d'abord fait mon passeport, mais mon contrat s'est expiré. Il ne me restait que mon passeport; et j'ai décidé de me rendre à Casablanca, à l'Office National de l'Immigration $[\ldots]$. 
Je suis donc allé en France à Arras, j'étais seul et ne connaissais personne quand je suis arrivé là-bas [...]. Et j'ai rencontré un problème, les logements étaient sales dans la ville d'Arras [...]. Après quinze jours de travail, je suis allé voir mon patron pour lui dire que le travail ne me plaisait pas. J'étais coffreur maçon dans la société. En réalité c'était les logements qui ne me plaisaient pas. C'était des baraquements, des bidonvilles, les draps étaient sales, il n'y avait personne pour les laver [...]. Bref, j'ai dit au patron que je voulais partir. Il n'a pas voulu, mais moi je lui ai dit que c'était obligé. Il m'a donc envoyé à l'Office National de l'Immigration à Paris. L'ONI faisait des appels d'offres pour le travail et cherchait donc des travailleurs. Il me disait, tu préfères aller travailler dans cette ville ou dans cette autre ville, et moi j'avais choisi Angers à cette époque [...].

J'avais choisi Angers, car on m'avait parlé d'Angers comme une ville calme et tranquille. Je ne connaissais personne à Angers, j'avais choisi Angers parmi un tas d'autres villes proposées, un peu au hasard, il fallait en choisir une [...]. J'ai donc été envoyé à Angers pour travailler dans la société Brochard \& Gaudichet au mois de mai 1965. En septembre 1965, c'est à cette époque que les amis du village de Beni Ayatt m'ont rejoint. Ils étaient trois, je ne leur avais pas envoyé de contrats de travail. Ils m'avaient dit qu'ils allaient venir, aussi je leur avais donné mon adresse, dans les quais de Félix Faure.

Ils sont arrivés une matinée tôt avant que j'aille au travail, à $7 \mathrm{~h} 30$. J'allais justement aller travailler lorsque le taxi qui les transportait les déposa devant chez moi. Le concierge était venu me chercher pour me dire de répondre à des gens. Je suis sorti et je les avais trouvés tous les trois et je les ai fait entrer. On se racontait les nouvelles du village, en prenant un café. J'ai appelé mon chef pour lui dire que j'allais être occupé car j'avais reçu de la famille, des cousins qui voulaient travailler. Je les ai emmenés avec moi, chez Brochard \& Gaudichet, et il les a fait travailler aussitôt. Le chef m'avait donné ma journée pour que je reste avec eux et il m'avait payé cette journée [...].»

Le témoignage de Monsieur AS. L., permet de mieux comprendre comment sont arrivées à Angers les trois personnes suivantes de Beni Ayatt 4 mois après le premier arrivant. Nous avons interviewé l'une de ces trois personnes, Monsieur AR. H., 69 ans, à la retraite. Voici un passage de son récit :

" Comment je suis arrivé en France? Il y avait une grande entreprise à l'époque qui est venue à Beni Ayatt pour avoir des hommes pour travailler en France [...]. L'entreprise nous avait envoyé des contrats de travail et nous n'avions plus qu'à faire nos passeports. Le jour de l'obtention de nos passeports, l'entreprise française nous a dit qu'elle n'avait plus besoin de nous. Et les passeports étaient faits et nous restaient entre les mains.

Nous étions 56 personnes à être concernées. Nous nous sommes séparés et chacun a suivi sa route, jusqu'à ce que tous soient arrivés en France, très peu d'entre eux sont restés au Maroc... Le jour où nous sommes partis, nous avions pris un billet de Casablanca à Marseille. À Marseille, nous avions pris un billet pour Montélimar. On y a passé la nuit, au petit matin, nous sommes allés chez JA. M., un ami de Beni Ayatt qui était allé en France et qui vivait dans le coin dans une ville qui s'appelait "Rochefort". Nous y sommes restés 3 jours. Nous étions 5 à ce moment-là. Puis nous nous sommes séparés. Je suis allé avec deux de mes amis [YO. H. et BE. H., aujourd'hui décédé], nous 
n'étions donc plus que trois et les deux autres sont allés de leur côté [...]. Et là, nous avons repris le train pour la ville d'Angers chez AS. L., on nous avait dit qu'il vivait là-bas. Et nous y sommes allés [...].

Pourquoi Angers? En fait, nous possédions deux adresses de deux de nos amis, l'un de Beni Ayatt, AS. L. et un autre homme d'Ait Attab qu'on appelait à l'époque "le vieux"... Quand on était à la gare de Montélimar, on a demandé laquelle des deux villes était la plus proche. Et l'on nous a répondu que c'était Angers, on a donc pris trois billets pour Angers... Le matin très tôt, nous sommes arrivés chez AS. L., qui nous a accompagnés, que Dieu le remercie encore. Il a demandé à son patron de nous embaucher et il l'a fait..."

AS. L est le premier arrivant en mai 1965. Puis les trois personnes suivantes arrivent en septembre, seulement avec des passeports et sans contrat de travail : YO. H, AR. H, EL. H. Ce dernier s'étant, à Montélimar, séparé d'autres compagnons partis également de Beni Ayatt. En octobre 1965, c'est une personne originaire de Beni Ayatt qui vient seule et va directement chez Monsieur AR. H qui lui trouve du travail.

En 1966, AS. L envoie un contrat de travail à l'un de ses cousins, HA.H, qui, plus tard en 1973, aide à son tour son frère HA. M. Puis, A.S. L fait aussi venir son frère en 1973 : AS. M. De son côté, AR. H a envoyé des contrats à son cousin EC. L en 1966, à AC. M en 1970, à BA. M en 1973 un autre de ses cousins, et ses deux frères AR. L en 1970 et AR. A en 1973. Puis, AC. M fait venir ses frères AC. L et AC. S en 1971, et EC. L contribue à la venue de ses trois frères EC. Z en 1969, EC. S et EC. M en 1971. D'autres personnes primomigrantes ont pu venir soit seules avec des passeports et des visas touristiques, et grâce à l'aide de l'un des membres de la communauté, ils ont trouvé du travail et un logement. C'est ainsi qu'entre 1965 et 1973, la communauté masculine de Beni Ayatt s'installe sur la ville d'Angers.

\section{Présentation de l'espace d'étude, Beni Ayatt au Maroc}

Beni Ayatt se situe à $25 \mathrm{~km}$ de la ville de Beni Mellal, dans le Moyen Atlas. La commune se trouve dans la province d'Azilal, dans la région TadlaAzilal. La commune de Beni Ayatt s'étend sur une superficie de $430 \mathrm{~km}^{2}$, située à cheval sur la grande plaine de Tadla et la chaîne montagneuse de l'Atlas Central. La zone de montagne est très déficitaire en eau, les quelques sources qu'elle compte sont d'un très faible débit et ne profitent qu'à une infime partie des habitants de cette région. Tous les types de milieux se rencontrent dans la commune : la terre fertile, la terre semi-aride, la terre aride, la montagne dénudée, la montagne couverte, la montagne farouche, les pâturages. Au pied même de cette montagne s'étend la commune de Beni Ayatt s'accrochant aux pentes basses et s'étalant sur la plaine en une profusion de vergers : c'est le grand périmètre des irrigations du Tadla.

La commune de Beni Ayatt n'est constituée que d'une seule tribu, celle des Beni-Ayatt, ancrée depuis fort longtemps, plus de deux siècles, tout au long de la montagne qui constitue le dir ou le piémont de la chaîne atlassique entre les Aït Bouzid (Afourer au nord et les Oulad Ayad au sud). La 
population s'étend sur la plaine de Tadla depuis la création des périmètres irrigués et grâce aux remembrements des terres qui ont suivi. Selon le recensement de la population de 1994, la population de la commune rurale de Beni Ayatt est de 19812 habitants et ne cesse d'augmenter (17168 habitants en 1982). Cette population est à dominante rurale et les principales activités des habitants sont l'agriculture et l'élevage. Les exploitations sont familiales et à caractère vivrier. L'habitat dans la plaine reste dispersé à certains endroits et dans certains douars de la commune, mais on peut qualifier l'habitat de Lkhémis, le principal centre, d'habitat groupé. Dans les années 1960, un grand mouvement de déplacement des habitants a été observé vers la plaine irriguée. Ainsi, on constate que la plupart des agriculteurs habitant cette plaine irriguée ont un foyer vide sur les plateaux ou en montagne.

\section{La répartition des Aït Ayad sur Angers}

\section{L'évolution spatiale des Marocains à Angers : d'une centralité à une périphérisation résidentielle}

À Angers, dans les années 1960, la population étrangère se localisait essentiellement dans les quartiers périphériques comme ceux des ZUP Nord et Sud. Cependant les quartiers centraux et péri-centraux connaissaient aussi un important taux de résidents étrangers en particulier autour du quartier de la gare. À partir de données de l'INSEE, on peut repérer la localisation de la population étudiée.

Un quartier se détache nettement des autres par la concentration des Aït Ayad. En effet, le quartier de la Roseraie semble être le quartier privilégié de cette communauté. C'est surtout la première génération qui y réside avec 15 familles, mais aussi 5 familles vivent sur le quartier de Monplaisir, essentiellement dans les rues encadrant la place de l'Europe (boulevard Schuman, boulevard Monplaisir...).

La seconde génération semble être mieux répartie sur l'ensemble de la ville. Bien que la majorité de cette catégorie se retrouve encore une fois sur la Roseraie avec une vingtaine de ménages. On peut remarquer toutefois, que des familles sont installées dans les quartiers Saint-Léonard, Justices, Grand Pigeon, Monplaisir, Verneau, Belle-Beille, Lac de Maine, et un couple dans le quartier Saint Serge. Une meilleure répartition dans l'espace angevin est donc constatée pour cette seconde génération. Cependant, cette population reste concentrée sur les quartiers périphériques. Le centre est peu habité par la population étrangère tandis que la Roseraie reste le quartier le plus occupé par les populations étrangères. Les Aït Ayad n'échappent pas à ce schéma. On peut se demander si c'est l'effet d'un regroupement voulu par la communauté ou est-ce une ségrégation spatiale subie par les populations étrangères en général? 


\section{Création d'un nouvel espace angevin par les Aüt Ayad}

Intéressons-nous d'abord au lieu de prière, la mosquée d'Angers. Nous nous sommes entretenus avec M. BA, imam de la mosquée d'Angers, et faisant partie des Aït Ayad, qui nous a éclairés sur différentes questions concernant le lieu de prière des musulmans à Angers. Nous avons appris qu'avant 1982, la communauté musulmane priait dans un local - anciennement le café " bon coin "- de la rue des Banchais. Ce local possédait deux niveaux : une salle en haut et une en bas. C'est à partir des années 1980 qu'une scission s'est produite entre les Turcs et les Maghrébins. Les Turcs ont décidé de prier dans la salle du bas et les Maghrébins à l'étage. En 1982, il n'y avait plus assez de place dans le local de la rue des Banchais une nouvelle mosquée est créée rue Saint-Exupéry. Le lieu de prière des musulmans suit les lieux de résidence, c'est-à-dire un éloignement du centre ville, une périphérisation.

Le marché du quartier Monplaisir est très fréquenté par la population maghrébine d'Angers. En effet, beaucoup d'achats des Aït Ayad y sont faits, mais aussi par toute la population maghrébine d'Angers en général. C'est un lieu très cosmopolite, fréquenté par des personnes de toutes origines culturelles. Mais bien plus qu'un lieu d'achat, ce marché est un véritable lieu de rencontre et de sociabilité pour les habitants maghrébins et d'origine maghrébine. Nous avons pu constater que la communauté utilisait cet espace pour faire des quêtes d'argent. C'est en effet le lieu qui permet de rencontrer pratiquement toute la communauté masculine et une partie des femmes aussi. Ainsi, s'il y a des nouvelles importantes du pays, c'est sur ce marché que se diffuse l'information. Ces différents espaces sont donc des lieux stratégiques qui agissent sur l'évolution du réseau migratoire.

Comme la population étrangère, les boucheries hâlales se localisent surtout à la périphérie de la ville. Elles ne vendent pas seulement de la viande hâlale, mais aussi des produits traditionnels rappelant ceux du pays, qu'on ne peut pas trouver dans d'autres épiceries. Il existe trois boucheries maghrébines sur Angers : une sur l'avenue Pasteur au sud de Monplaisir, une autre dans le quartier du Grand Pigeon, et enfin une troisième dans le quartier Saint-Léonard. C'est tout récemment (avril 2002) qu'une quatrième boucherie tenue par un Marocain, s'est installée dans le quartier de la Roseraie, place Jean Vilar.

Ainsi, nous pouvons observer une réelle périphérisation de la population des Aït Ayad sur l'espace d'arrivée, autant dans ses lieux d'habitat que dans sa pratique de l'espace. Qu'en est-il de son espace d'origine?

\section{Évolution de l'espace de départ par la migration et la circulation migratoire}

\section{Impact spatial dans l'espace de départ}

Nous n'allons pas détailler les investissements des migrants à Beni Ayatt mais plutôt essayer de comprendre comment les Aït Ayad réoccupent 
l'espace de départ. Plus l'émigration y est forte, plus les constructions sont nombreuses, en particulier le long des axes routiers et autour des centres ruraux, qui sont généralement des souks. Outre les maisons d'habitation, les migrants investissent aussi dans des petits commerces (épiceries, cafés...), et appuient certains projets (mosquées) par leur participation financière, et même certains projets de développement comme la réalisation de petits sentiers, la fréquence de l'arrivée en eau dans le village...

Ainsi, une évolution sur le paysage et l'espace de Beni Ayatt peut être observée depuis les années 1960. En effet, on note un glissement des populations des montagnes du sud de Beni Ayatt, vers la plaine, au nord. Nous avons pu remarquer lors de nos séjours au Maroc des maisons abandonnées dans le village de Tizgui. Elles l'ont été par des immigrés mais pas seulement. C'est depuis quelques années seulement que Lkhémis est devenue le douar central de Beni Ayatt. Toutes les infrastructures, aussi minimes soient-elles, se concentrent à Lkhémis. Ce rapprochement de la route et du centre administratif permet de bénéficier des groupes électrogènes communaux, de la distribution d'eau potable, des moyens de télécommunication, des établissements sociaux, mais aussi de profiter d'une topographie plane où le migrant peut accéder facilement en voiture. Par ailleurs, ce glissement remarquable des logements des migrants vers le petit centre de Lkhémis, a pour conséquence évidente d'accentuer la " micro-urbanisation " de Lkhémis. L'immigration a donc enclenché un processus d'urbanisation à Lkhémis.

Désormais, c'est là que tous les services (dispensaire, tribunal, etc..) et commerces de Beni Ayatt se concentrent. Il est donc évident que les migrants en choisissant de se réinstaller dans leur commune d'origine, vont choisir le lieu le plus adapté, où ils pourront disposer d'un minimum de confort, c'est-à-dire de l'eau, de l'électricité...

\section{À générations différentes, investissements différents}

Ce sont surtout les migrants de la première génération qui investissent à Beni Ayatt dans l'habitat, dans des projets commerciaux, ou aident à la construction de mosquées... Les choses sont différentes pour la seconde génération.

"Crépuscule " est une association loi 1901 qui a son siège à Angers. Les adhérents sont d'origines et d'âges divers. Un de leurs récents projets est la création d'une bibliothèque à Beni Ayatt. Le groupe qui s'investit dans ce projet est formé d'une dizaine de jeunes dont certains sont issus de l'immigration et issus du groupe des Aït Ayad. Pourquoi ces jeunes veulent-ils s'investir à Beni Ayatt? C'est une question que je leur ai posée. Nadia répond qu'elle allait souvent en vacances dans le village d'origine de ses parents, et qu'elle a voulu faire quelque chose d'utile pour ce village, quelque chose qui pourrait créer des liens entre leur association et des jeunes de Beni Ayatt. Une association (" Tifaouine " en berbère, "Aube " en 
français) a donc été créée à Beni Ayatt par les jeunes Marocains, qui aura pour objet de gérer cette bibliothèque. C'est un véritable échange culturel qui s'est développé entre "Crépuscule " et "Tifaouine " (un documentaire de 52 minutes retrace le projet). Cette initiative a été très bien accueillie par la population de Beni Ayatt et par les jeunes du village. Il a permis de donner une autre image des jeunes issus de l'immigration aux Marocains. Il faut noter qu'aujourd'hui, les autorités marocaines encouragent le retour des jeunes Marocains résidant à l'étranger et qui souhaitent travailler au développement de leur région d'origine.

Nous pouvons noter un décalage par rapport à la forme d'investissement dans la commune de Beni Ayatt entre les primo-migrants et les générations suivantes. La première génération investit dans le développement territorial de sa commune d'origine, avec des préoccupations individuelles, religieuses, économiques, financières... Alors que ces nouvelles générations ont une préoccupation moins individuelle et plus collective, ils n'investissent pas dans l'espace d'origine de leurs parents, ils s'investissent d'une manière plus personnelle.

\section{L'impact de la migration dans le village d'origine : un développement pour la commune?}

D'après El Hamraoui ${ }^{2}$, la migration est un phénomène bénéfique. Elle constitue un facteur de développement des pays pauvres. Elle permet de renflouer leur balance des paiements et de financer les achats indispensables de produits de première nécessité et de biens d'équipement. Elle est source d'investissements dans le pays d'origine, permet d'atténuer l'intensité du chômage et de réduire la densité démographique et le surpeuplement. La migration contribue fortement à l'amélioration du niveau de vie et à l'épanouissement du migrant. Mais sur le plan social, les effets bénéfiques de la migration semblent se suspendre avec la migration. En effet, la migration est un facteur d'entretien passager, qui permet une promotion rapide mais aussi une chute non moins rapide à l'issue éventuelle de la migration.

On peut penser que les initiatives des migrants de Beni Ayatt se limitent trop à des projets personnels et individuels qui ne mènent pas à la réalisation d'un vrai projet de développement. Néanmoins, si ces initiatives étaient partagées par tous, avec des appuis financiers des banques et de l'État, des projets de développement économique pourraient voir le jour. Par ailleurs, le fait que le premier investissement des migrants dans leur commune d'origine soit la construction d'une maison d'habitation peut révéler la volonté d'un certain retour. Mais qu'en est-il dans la réalité?

2. El Hamraoul, Abdelhamid, Le phénomène migratoire et son impact sur la société et l'espace dans le Rif, Urbana, Tours, 1998. 


\section{L'émigration ayattoise, créatrice d'un espace humain angevin et « reterritorialisation"de Beni Ayatt : \\ l'« espace de l'entre deux»}

Trois formes d'espaces sont mis en relation. L'espace de départ, Beni Ayatt, a d'abord connu une déterritorialisation avec le départ de migrants vers l'Europe et essentiellement vers Angers. Puis la ville d'Angers va connaître une reterritorialisation avec l'arrivée d'un grand nombre de migrants dans les années 1960, à travers les flux financiers, de retraite, professionnel, touristique, socio-culturel. Toute cette circulation va modifier l'espace angevin avec la création d'un nouvel espace migratoire sur Angers. La migration des Aït Ayad a aussi joué un rôle sur le changement spatial de l'espace de départ. Ainsi, les flux financiers et les impacts des Aït Ayad de retour à Lkhémis avec un certain nombre de constructions a permis la reterritorialisation du centre de Beni Ayatt.

Nous pouvons noter une autre contradiction dans la dualité de ces deux espaces. Il existe une réelle périphérisation de l'espace angevin des Aït Ayad autant dans la localisation, dans la pratique ou dans la création de nouvelles infrastructures et donc de nouveaux espaces. Au contraire, si l'on regarde le nouveau territoire de Beni Ayatt créé par les migrants angevins, c'est la centralité de l'espace qui est occupée par ces derniers. L'habitat des Aït Ayad ainsi que la création des commerces se localisent pratiquement tous dans le centre de Beni Ayatt, à Lkhémis. Et dans le douar central, cette localisation se précise le long de la route principale qui est le cœur de Lkhémis. Ces espaces sont-ils révélateurs d'un comportement et d'une situation identitaire du migrant, c'est-à-dire " périphériques " en France et " centraux " au Maroc?

Mais c'est aussi dans la dualité de ces espaces et dualité de ce positionnement social et identitaire du migrant que se situe la circulation migratoire et qu'on pourra peut-être un jour mettre un contenant à ce " contenu de l'entre deux". 


\section{RESUME}

Cette étude tente de comprendre les migrations qui existent entre le Maroc et la France depuis les années 1960. Elle s'intéresse particulièrement à une région précise du Maroc : Beni Ayatt dans le Moyen Atlas. L'émigration maghrébine vers l'Europe est une conséquence du phénomène colonial qui a favorisé la mobilité interne et externe des Maghrébins. Après la Seconde Guerre mondiale, tout a été mis en place pour constituer l'espace migratoire de cette population. Ainsi, après les indépendances de ces pays, des mutations sociales et spatiales ont accentué la migration vers l'Europe. Dans les années soixante des filières migratoires se mettent en place grâce à des réseaux familiaux, villageois et à un appel de main-d'œuvre par les pays d'accueil comme la France. C'est ainsi que s'est constitué le champ migratoire Beni Ayatt/Angers. Cette filière migratoire se composait essentiellement d'hommes venus travailler en France de façon régulière. Puis le regroupement familial a permis la venue des femmes et des enfants ainsi, que l'installation définitive de ces familles.

\section{ABSTRACT}

This study is an attempt to understand the migratory streams between Morocco and France since the sixties. I'm particularly interested in a definite Moroccan region: Beni Ayatt in the Middle Atlas Mountains. The North African emigration towards Europe is a consequence from the colonial phenomenon, which has contributed to the domestic and foreign mobility of North African people. After the Second World War, all was done to create a migratory space for this population. This is the reason why, after the independency given to those countries, social and space transformation have increased the migration towards Europe. During the sixties, migratory networks were created thanks to family organisations and to an appeal to foreign labour by reception countries like France. That is how the migratory space Beni Ayatt/Angers was formed. This migratory network was essentially composed of men who had come to work regularly in France. Afterwards the family regrouping made possible for women and children to come, which gave them the opportunity to settle permanently. 